\title{
Centralized Processing of multiple smartphone raw measurements with fixed and known position onboard a vehicle
}

\author{
Arnau Ochoa Banuelos, CS Group \\ Jan Bolting, Xiao Hu, Paul Thevenon, ENAC, Université de Toulouse \\ Bryan Cazabonne, Matthieu Pascaud, Arnaud Ginestet CS Group
}

\section{BIOGRAPHY}

Arnau Ochoa Banuelos graduated as telecommunications engineer and computer engineer from Universitat Autònoma de Barcelona (UAB) in 2019. He is currently pursuing a MSc in navigation and space telecommunications.

Jan Bolting biography text goes here (50 words or less).

Xiao Hu graduated as electronic engineer in digital communications and received the M.S. double degree in Navigation and Telecommunications from the French Civil Aviation University (ENAC), in Toulouse, France, in 2017. Since 2017, he is a Ph.D. student within the SIGnal processing and NAVigation (SIGNAV) research group of the TELECOM laboratory inside ENAC. His current research interests focus on Precise GNSS Positioning and hybridization of GNSS with other sensors.

Paul Thevenon graduated as electronic engineer from Ecole Centrale de Lille in 2004 and obtained in 2007 a research master at ISAE in space telecommunications. In 2010, he obtained a $\mathrm{PhD}$ degree in the signal processing laboratory of ENAC in Toulouse, France. From 2010 to 2013, he was employed by CNES, the French space agency, to supervise GNSS research activities and measurement campaigns. Since the July 2013, he is employed by ENAC as Assistant Professor. His current activities are GNSS signal processing, GNSS integrity monitoring and hybridization of GNSS with other sensors.

Bryan Cazabonne biography text goes here (50 words or less).

Matthieu Pascaud biography text goes here (50 words or less).

Arnaud Ginestet biography text goes here (50 words or less).

\begin{abstract}
The capacity to obtain precise positioning using smartphones has become conceivable in recent years thanks to the release of multi-constellation, multi-frequency smartphones. Furthermore, from 2016 GNSS raw measurements are made available to developers on Android smartphones. For these reasons, and considering that smartphones have access to the Internet, it is plausible to aim at implement decimeter-level positioning techniques on these devices. Nonetheless, smartphones also have some flaws when using them as GNSS receivers. Firstly, the GNSS antenna and the rest of the electronics are embedded inside the body of the smartphone. This causes the signals to be received at a low signal-to-noise ratio and can cause small interference that further degrade the received signal. Secondly, smartphone manufacturers usually put low-cost GNSS chips in their smartphones since the manufacturing cost of the device comprises many more components. Therefore, the quality of the hardware cannot be compared to that of the high-end GNSS receivers that are commonly used for high-accuracy positioning applications.

The objective of this project is to obtain the best accuracy from a set of GNSS measurements that are obtained with smartphones. This project is comprised within the Google Smartphone Decimeter Challenge. For this competition, the Android GNSS team released a pool of datasets containing GNSS and inertial measurements obtained with smartphones on different locations around the San Francisco Bay Area.

This article presents the solution that has been implemented for this competition. One interesting characteristic of the data published by Google is that many trajectories contain measurements from more than one phone. For this reason, we decided to combine the measurements from multiple receivers in an RTK implementation. This method allows estimating the position, velocity, and attitude of the vehicle while at the same time improving the accuracy of the positioning.
\end{abstract}




\section{INTRODUCTION}

In May 2016, Google announced the availability of GNSS raw measurements on smartphones with the release of Android 7. From then, newer versions have been improving the availability of this data and have also included access to more information such as the Automatic Gain Control (AGC) or the code type of the signal. Newer phones have also added compatibility with other constellations apart from GPS with some devices supporting up to 5 different systems, namely GPS, GLONASS, Galileo, BeiDou and QZSS. Furthermore, thanks to the decreasing cost and the miniaturization of the hardware, some manufacturers included dual frequency chips on their higher-end products, the first being the Xiaomi Mi 8 which was launched on May 2018. During all these years, the interest on the Android GNSS raw measurements has been steadily increasing [1].

This year 2021, Android's GNSS team has launched the Google Smartphone Decimeter Challenge, which will be presented at ION GNSS+ 2021. For this competition, Google released a pool of 48 datasets containing inertial and GNSS raw measurements obtained from five different smartphones. These datasets were collected on highways and urban areas in the San Francisco Bay Area. In addition, State Space Representation (SSR) corrections and Observation Space Representation (OSR) corrections are provided from SwiftNavigation Inc. ${ }^{1}$ and Verizon Inc. ${ }^{2}$. The goal of this competition is to develop an algorithm capable of obtaining the best possible accuracy on these datasets, setting decimeter-level positioning as an accuracy target. In addition, another pool of 73 datasets is provided which follow the same structure but also contain ground-truth files collected by a high-precision NovAtel system. Table 1 shows an overview of the train and test datasets that are provided for this competition. The score is computed by taking the mean between the 50 and the 95 percentiles of the horizontal error of each trace and then averaging this mean for all traces. This is

$$
s=\operatorname{mean}\left\{s_{1}, s_{2}, \ldots, s_{48}\right\} \quad \mid s_{i}=\operatorname{mean}\left\{P_{50, t}, P_{95, t}\right\}
$$

where $s$ is the final score in meters, and $P_{50, t}, P_{95, t}$ are the 50 and 95 percentiles of the horizontal error for the trace $t$. In this paper, the individual scores $s_{i}$ obtained on each trace are used to compare the different solutions.

\begin{tabular}{|c|c|c|}
\hline Dataset & Train & Test \\
\hline Campaigns & 29 & 19 \\
\hline Traces & 73 & 48 \\
\hline \multirow{2}{*}{ Phones } & Pixel4, Pixel4Modded, & Pixel4XL, Pixel4XLModded, \\
& Pixel5, Mi8, SamsungS20Ultra & $\begin{array}{c}\text { Pixel4, Pixel4Modded, } \\
\text { Pixel4XL, Pixel4XLModded, } \\
\text { Pixel5, Mi8, SamsungS20Ultra }\end{array}$ \\
\hline Ground-truth & Yes & No \\
\hline
\end{tabular}

Table 1: Datasets of the Google Smartphone Decimeter Challenge

Different techniques have been long used to achieve high accuracy in the GNSS position computation. In order to achieve decimeter-level accuracy, the use of carrier phase measurements and the capability to obtain integer solutions for the ambiguities are two features that are required. The two solutions that are classically applied to solve this problem are RTK positioning and PPP. RTK is a technique that relies on differential GNSS measurements in order to resolve the carrier-phase ambiguities. This technique is based on the use of pseudorange and carrier-phase measurements that are obtained in a station whose location is well known. This method allows to eliminate the main sources of error in GNSS, but it can only be used in the vicinity the base station. However, a high-bandwidth communication channel is needed for real time applications, as the OSR data tends to be heavy. PPP provides an attractive alternative to differential GNSS techniques such as RTK. The PPP method uses un-differenced, multi-frequency, pseudorange and carrier-phase observations along with precise satellite orbit and clock products, which can provide centimeter-level precision. PPP provides some benefits with respect to the differential techniques, mainly due to the fact that no reference stations are needed. Furthermore, although some communication channel is required in order to retrieve the precise satellite orbit and clock products, less bandwidth is required in order to transmit SSR data compared to OSR data. However, PPP requires a long time of convergence in order to obtain an accurate float estimate of the carrier-phase ambiguities. In addition, this method also requires a cautious modeling and data screening for outliers and carrier-phase cycle slips, which is more challenging than for the differential GNSS approach. In this competition, RTK seems to be a more appropriate technique to obtain high precision positioning since OSR data is already provided which has been taken from stations that are less than $10 \mathrm{~km}$ away from the locations where the measurements were taken. Furthermore, since the smartphones have access to the Internet, it is plausible to apply this technique in a real-time application in the future.

The Google Smartphone Decimeter Challenge datasets have a particularity that our team wants to exploit: it involves several smartphones which are located at a fixed location onboard the vehicle during one data collect. As such, the multiple sensors

${ }^{1}$ WWw . Swiftnav.com/google-smartphone-decimeter-challenge

${ }^{2}$ webscope . sandbox . yahoo . com/catalog . php?datatype=s\&did=88\&guccounter $=1$ 
can be considered as an array of sensors, whose observations can be processed in a centralized manner. For example, by including the geometric relation between the different GNSS antennas of the multiple GNSS receivers, it is possible to establish an observation model that will include the attitude of the array in addition to its position.

This technique has been investigated previously by Xiao Hu et al. [2] [3] [4] in the context of RTK in an Extended Kalman Filter (EKF). It has proved to provide increased performance, in terms of cycle slip detection, solution availability, fixed ambiguity solution availability and accuracy, providing some attitude information as well. These benefits appear thanks to some kind of spatial diversity: because the antennas are separated spatially, they may not be affected by the same propagation events. This spatial diversity gain increases with the spatial separation of the antennas. Due to the small separation in the Google data collect (on the order of tenths of $\mathrm{cm}$ ), the effectiveness of this feature had to be verified. In this document, a multi-receiver RTK is compared to a single-receiver RTK and it is shown that the multi-receiver solution is almost always better than the single-receiver solution. This multi-receiver technique is based on the assumption that the geometry of the receivers is known. Although this geometry is not explicitly provided in the Google Smartphone Decimeter Challenge, it can be obtained from the ground-truth trajectories that are provided in the train dataset.

This paper focuses on the improvement in performance that can be obtained when combining GNSS measurements from multiple receivers. Section II describes the mathematical models of the methods that are compared in this paper. Then, Section 3 provides a brief analysis of the Android GNSS raw measurements. The results obtained from the single- and multi-receiver RTK are shown in Section IV, where they are also compared to standalone Single-Point-Position (SPP) and Single-Point-Position-and-Velocity (SPPV) solutions. Finally, the conclusions are drawn in Section V.

\section{MATHEMATICAL MODELS}

In this work, two RTK positioning models are developed, both based on Kalman Filters (KF). The first method combines the standard double-differenced (DD) pseudorange and carrier-phase measurements used in RTK as well as non-differenced Doppler shift, which provides velocity estimates that indirectly smooth the noise of the ranging measurements in the Kalman Filtering. The second method is based on the same principle as the first method but it combines the measurements from multiple receivers in an EKF, which allows the filter to estimate the attitude of the vehicle. In order to assess the viability of using RTK with the Android raw GNSS measurements, two standalone non-differential methods are used, namely a Single-Point-Position (SPP) EKF which uses pseudorange measurements and a Single-Point-Position-and-Velocity (SPPV) EKF which uses pseudorange and Doppler measurements. All these methods combine GPS L1/L5, Galileo E1/E5a and BeiDou B1I measurements in order to provide additional redundancy and better geometry. Furthermore, an outlier rejection technique has been applied in order to reduce multipath effects and provide some means of carrier-phase cycle-slip detection. This technique is based on a standard hypothesis testing applied to the innovations [5]. The KFs have been implemented sequentially in order to reduce the computational costs. This is especially important in the multi-receiver RTK, since it requires the processing of a larger number of measurements and the estimation the carrier-phase ambiguities for all the receivers, which dramatically increases the size of the covariance matrices. This section provides a description of the single-receiver and the multi-receiver RTK models.

\section{Single-Receiver RTK}

As previously stated, both RTK methods use double-differenced (DD) pseudorange and carrier-phase measurements as well as non-differenced Doppler shift. These measurements are modeled as follows

$$
\begin{gathered}
\rho_{j}^{D D}=r_{s, p}-r_{u, p}-r_{s, j}+r_{u, j}+\varepsilon_{\rho, j} \\
\phi_{j}^{D D}=r_{s, p}-r_{u, p}-r_{s, j}+r_{u, j}+\lambda_{j}\left(N_{p}^{S D}-N_{j}^{S D}\right)+\varepsilon_{\phi, j} \\
\dot{\rho}_{i}=\dot{r}_{i}+c\left(\dot{\delta} t_{u}+\dot{\delta} t_{I F}+\dot{\delta} t_{I S}\right)+\varepsilon_{\dot{\rho}_{i}}
\end{gathered}
$$

where:

- $\rho_{j}^{D D}$ refers to the $j$-th DD pseudorange

- $\phi_{j}^{D D}$ refers to the $j$-th DD carrier-phase

- $\dot{\rho}_{i}$ refers to the $i$-th pseudorange rate (i.e. the Doppler shift expressed in meters per second), which has been corrected by the satellite's clock drift

- $r_{a, b}$ denotes the true range between receiver $a$ and satellite $b$

- Subindices $s$ and $u$ denote the base station and the user receivers, respectively

- Subindices $p$ and $j$ denote the pivot and the $j$-th satellites, respectively 
- $N_{a}^{S D}$ refers to the single-differenced carrier-phase ambiguity for satellite $a$

- $l a m b d a_{j}$ is the carrier wavelength of signal $j$

- $\varepsilon_{\rho, j}, \varepsilon_{\phi, j}$ and $\varepsilon_{\dot{\rho}_{i}}$ refer to the residual errors of the $j$-th DD pseudorange, $j$-th DD carrier-phase and $i$-th pseudorange rate, respectively

- $\dot{\delta} t_{u}, \dot{\delta} t_{I F}$ and $\dot{\delta} t_{I S}$ refer to the user, inter-frequency and inter-system clock drifts, respectively

- Subindex $j$ denotes the DD measurement and is in the range $1, \ldots, N_{D D}$

- Subindex $i$ denotes the non-differenced measurement and is in the range $1, \ldots, N_{\text {meas }}$

- $c$ is the speed of light

It is worth noting that two different approaches can be followed regarding the ambiguities in equation (3). One approach is to join $N_{p}^{S D}-N_{j}^{S D}$ as double-differenced ambiguities and include them into the state vector. However, following this approach, every time the pivot satellite changes all the DD ambiguities need to be re-estimated. This approach can be interesting to implement for applications in which the reception of GNSS signals is very stable and there is not much signal blocking. However, when this is not the case, it may be more interesting to keep track of the single-differenced ambiguities and compute the double differences each time. Although this approach is a bit more complex in the implementation, it is the one selected for this project as it has been seen that the GNSS measurements obtained from smartphones often lose track of the signals.

Every time a new signal is acquired, either because a new satellite is in view or because the receiver has reacquired lock with the signal, an initial estimation of the ambiguity has to be obtained. In order to obtain these initial estimations, the Code-Minus-Carrier (CMC) is used.

$$
\begin{gathered}
C M C_{i}^{S D}=\rho_{i}^{S D}-\phi_{i}^{S D}=-\lambda N_{i}^{S D}+\varepsilon_{\rho, i}^{S D}-\varepsilon_{\phi, i}^{S D} \\
\hat{N}_{i}^{S D}=-\frac{C M C_{i}^{S D}}{\lambda}
\end{gathered}
$$

where $\hat{N}_{S D, i}$ is the estimated single-differenced ambiguity. In the following, the state and the measurement vectors are described along with the state-transition and measurement models.

\section{State transition model}

In our KF modeling, the state vector contains the user-station baseline vector $\boldsymbol{b}_{u s}$, the user velocity $\dot{\boldsymbol{u}}$, the aforementioned clock drifts, and the SD ambiguities.



where $\dot{\boldsymbol{\delta}} \boldsymbol{t}_{I F}$ and $\dot{\boldsymbol{\delta}} \boldsymbol{t}_{I S}$ are vectors of size $N_{\text {freq }}-1$ and $N_{\text {sys }}-1$, respectively. $\boldsymbol{N}^{S D}$ is a vector containing the SD ambiguities of all the common satellites between the receiver and the station. Since this project uses multiple receivers and multiple frequency bands, one pivot satellite has to be selected for each pair of constellation and frequency; therefore $N^{S D}$ is of size $N_{D D}=N_{\text {meas }}-N_{\text {piv }}$, where $N_{\text {meas }}$ is the number of carrier phase measurements available and $N_{\text {piv }}$ is the number of pivot satellites (i.e. number of constellation-frequency pairs).

The state transition model is defined as an integration of the velocity for the user-station baseline vector and a random-walk model for the rest of state parameters:

$$
\begin{aligned}
\boldsymbol{b}_{u s_{n}} & =\boldsymbol{b}_{u s_{n-1}}+\dot{\boldsymbol{u}}_{n-1} \Delta t \\
\dot{\boldsymbol{u}}_{n} & =\dot{\boldsymbol{u}}_{n-1}+\boldsymbol{w}_{\dot{u}, n} \\
c \dot{\delta} t_{u, n} & =c \dot{\delta} t_{u, n-1}+w_{c \dot{\delta} t_{u}, n} \\
c \dot{\boldsymbol{\delta}} \dot{\boldsymbol{t}}_{I F, n} & =c \dot{\boldsymbol{\delta}} t_{I F, n-1}+\boldsymbol{w}_{c \dot{\delta} t_{I F}, n} \\
c \dot{\boldsymbol{\delta}} \dot{\boldsymbol{t}}_{I S, n} & =c \dot{\boldsymbol{\delta}} t_{I S, n-1}+\boldsymbol{w}_{c \dot{\delta} t_{I S, n}} \\
\boldsymbol{N}_{n}^{S D} & =\boldsymbol{N}_{n-1}^{S D}+\boldsymbol{w}_{N S D, n}
\end{aligned}
$$


where

$$
\begin{aligned}
\boldsymbol{w}_{\dot{u}, n} & \sim \mathcal{N}\left(0, \sigma_{\dot{u}}^{2} \boldsymbol{I}_{3}\right) \\
w_{c \dot{\delta} t_{u}, n} & \sim \mathcal{N}\left(0, \sigma_{c \dot{t_{u}}}^{2}\right) \\
\boldsymbol{w}_{c \dot{\delta} t_{I F}, n} & \sim \mathcal{N}\left(0, \sigma_{c \dot{\delta} t_{I F}}^{2} \boldsymbol{I}_{N_{f r e q}-1}\right) \\
\boldsymbol{w}_{c \dot{\delta} t_{I S}, n} & \sim \mathcal{N}\left(0, \sigma_{c \dot{\delta} t_{I S}}^{2} \boldsymbol{I}_{N_{s y s}-1}\right) \\
\boldsymbol{w}_{N S D, n} & \sim \mathcal{N}\left(0, \sigma_{N S D}^{2} \boldsymbol{I}_{N_{D D}}\right)
\end{aligned}
$$

Then, the state transition matrix can be written as

$$
\boldsymbol{F}=\left[\begin{array}{ccccc}
\boldsymbol{I}_{3} & \Delta t \boldsymbol{I}_{3} & 0 & \cdots & 0 \\
\mathbf{0} & 1 & 0 & \cdots & 0 \\
\vdots & & \ddots & & \vdots \\
0 & \cdots & 0 & 1 & 0 \\
0 & \cdots & 0 & 0 & 1
\end{array}\right]
$$

and the corresponding process noise matrix $Q$ is given by the diagonal matrix

$$
\boldsymbol{Q}=\operatorname{diag}\left(\left[\begin{array}{lllllll}
\mathbf{0}_{1 \times 3} & \sigma_{\dot{u}}^{2} \mathbf{1}_{1 \times 3} & \sigma_{c \dot{\delta} t_{u}}^{2} & \sigma_{c \dot{t_{I F}}}^{2} \mathbf{1}_{1 \times\left(N_{f r e q}-1\right)} & \sigma_{c \dot{\delta} t_{I S}}^{2} \mathbf{1}_{1 \times\left(N_{s y s}-1\right)} & \sigma_{N S D}^{2} \mathbf{1}_{1 \times N_{D D}}
\end{array}\right]\right)
$$

\section{Measurement model}

Considering the distance between the receiver and the station to be small enough, the double difference of the geometric ranges in equations (2) and (3) can be rewritten as the difference of the Line-Of-Sight (LOS) vectors between the station and the two satellites, projected onto the baseline vector between station and receiver [6]. Here, the subscript $n$ denoting the epoch is dropped in order to simplify the notation.

Then, the measurement equation for the DD pseudorange is

$$
\boldsymbol{h}_{\rho^{D D}}(\boldsymbol{x})_{[j]}=\left(\boldsymbol{e}_{s, p}-\boldsymbol{e}_{s, j}\right)^{T} \boldsymbol{b}_{u s}
$$

where $e_{s, p}$ and $e_{s, j}$ are the LOS unit vectors from the station to the pivot and non-pivot satellites, respectively. Similarly, the measurement equation for the DD carrier phase is

$$
\boldsymbol{h}_{\phi^{D D}}(\boldsymbol{x})_{[j]}=\left(\boldsymbol{e}_{s, p}-\boldsymbol{e}_{s, j}\right)^{T} \boldsymbol{b}_{u s}+\lambda\left(N_{p}^{S D}-N_{j}^{S D}\right)
$$

Regarding the pseudorange rate, the measurement function can be obtained from the equation (4).

$$
\boldsymbol{h}_{\dot{\rho}}(\boldsymbol{x})_{[i]}=\left(\dot{\boldsymbol{s}}_{i}-\dot{\boldsymbol{u}}\right)^{T} \boldsymbol{e}_{i}+c \dot{\delta \delta} t_{u}+c \dot{\delta} t_{I F, i}+c \dot{\delta \delta} t_{I S, i}
$$

From these three equations the measurement matrix is defined as

$$
\boldsymbol{H}=\left[\begin{array}{c}
\boldsymbol{H}_{\rho^{D D}} \\
\boldsymbol{H}_{\phi^{D D}} \\
\boldsymbol{H}_{\dot{\rho}}
\end{array}\right]
$$

where

$$
\boldsymbol{H}_{\rho^{D D}[j:]}=\left[\begin{array}{lllll}
\left(\boldsymbol{e}_{s, p}-\boldsymbol{e}_{s, j}\right)^{T} & \mathbf{0}_{1 \times 3} & \mathbf{0}_{1 \times N_{f r e q}-1} & \mathbf{0}_{1 \times N_{s y s}-1} & \mathbf{0}_{1 \times N_{D D}}
\end{array}\right]
$$




$$
\begin{aligned}
& \boldsymbol{H}_{\phi^{D D}[j:]}=\left[\begin{array}{lllllllll}
\left(\boldsymbol{e}_{s, p}-\boldsymbol{e}_{s, j}\right)^{T} & \mathbf{0}_{1 \times 3} & \mathbf{0}_{1 \times N_{f r e q}-1} & \mathbf{0}_{1 \times N_{s y s}-1} & \lambda & 0 & \cdots & 0 & -\lambda
\end{array}\right] \\
& \boldsymbol{H}_{\dot{\rho}[i:]}=\left[\begin{array}{lllll}
\mathbf{0}_{1 \times 3} & \left(\boldsymbol{e}_{s, p}-\boldsymbol{e}_{s, j}\right)^{T} & \boldsymbol{a}_{i}^{T} & \boldsymbol{b}_{i}^{T} & \mathbf{0}_{1 \times N_{s a t}}
\end{array}\right]
\end{aligned}
$$

with the $\lambda$ and $-\lambda$ in (17) located at the columns that correspond to the pivot and non-pivot satellites, respectively. Vectors $\boldsymbol{a}_{i}$ and $\boldsymbol{b}_{i}$ contain a 1 at the column that corresponds to the frequency and the constellation of the $i$-th measurement, respectively. Subscripts $[j:]$ and $[i:]$ denote the $j$-th and the $i$-th matrix rows, respectively.

The measurement covariance matrix for the DD measurements can be obtained from the covariance matrices of SD measurements. Considering that the noise of the receiver measurements is independent from that of the station measurements, the SD measurement covariance matrices can be obtained by simple addition of the measurement covariances of the two sources. Assuming the measurement covariances to be the same in the receiver and in the station, the SD measurement covariance matrices can be defined from the covariance matrices of the smartphone measurements. These are built using the uncertainty parameters included in the Android GnssLog files. Then, the SD measurement covariance matrices are

$$
\begin{aligned}
& \boldsymbol{R}_{\rho^{S D}}=2 \boldsymbol{R}_{\rho} \\
& \boldsymbol{R}_{\phi^{S D}}=2 \boldsymbol{R}_{\phi}
\end{aligned}
$$

The DD measurement covariance matrices are then given by

$$
\begin{aligned}
\boldsymbol{R}_{\rho^{D D}} & =\boldsymbol{D} \boldsymbol{R}_{\rho^{S D}} \boldsymbol{D}^{T} \\
\boldsymbol{R}_{\phi^{D D}} & =\boldsymbol{D} \boldsymbol{R}_{\phi^{S D}} \boldsymbol{D}^{T}
\end{aligned}
$$

where $\boldsymbol{D}$ is the double-differencing matrix where the location of the column full of 1's correspond to the pivot satellite and the location of the -1's matches the position of the non-pivot satellite:

$$
\boldsymbol{D}=\left[\begin{array}{ccccc}
-1 & 0 & \cdots & 0 & 1 \\
0 & -1 & \cdots & 0 & 1 \\
\vdots & \vdots & \ddots & \vdots & \vdots \\
0 & 0 & \cdots & -1 & 1
\end{array}\right]
$$

The resulting covariance matrices $\boldsymbol{R}_{\rho^{D D}}$ and $\boldsymbol{R}_{\phi^{D D}}$ are non-diagonal, which results in a non-diagonal measurement covariance matrix

$$
\boldsymbol{R}=\left[\begin{array}{ccc}
\boldsymbol{R}_{\rho^{D D}} & \mathbf{0}_{N_{D D}} & \mathbf{0}_{N_{D D} \times N_{s a t}} \\
\mathbf{0}_{N_{D D}} & \boldsymbol{R}_{\phi D D} & \mathbf{0}_{N_{D D} \times N_{s a t}} \\
\mathbf{0}_{N_{s a t} \times N_{D D}} & \mathbf{0}_{N_{s a t} \times N_{D D}} & \sigma_{\dot{\boldsymbol{\rho}}}^{2} \boldsymbol{I}_{N_{s a t}}
\end{array}\right]
$$

This non-diagonal matrix presents a problem when implementing a sequential Kalman Filter update [7]. One option to solve this issue is to perform a diagonalization of the resulting matrix by Cholesky decomposition, as it is explained in section 7.4 of [8]. This option was tested during the realization of this project but the accuracy of the position estimation was reduced. This is not expected considering that the inclusion of the non-diagonal terms in the covariance matrix improves the modeling of the noise, which should improve the performance of the algorithm. Given the limited time for this project, this issue was faced by simply considering a diagonal measurement matrix and inflating the DD noise.

$$
\boldsymbol{R}=\left[\begin{array}{ccc}
\sigma_{\rho^{D D}}^{2} \boldsymbol{I}_{N_{D D}} & \mathbf{0}_{N_{D D} \times N_{D D}} & \mathbf{0}_{N_{D D} \times N_{s a t}} \\
\mathbf{0}_{N_{D D} \times N_{D D}} & \sigma_{\phi D D}^{2} \boldsymbol{I}_{N_{D D}} & \mathbf{0}_{N_{D D} \times N_{s a t}} \\
\mathbf{0}_{N_{D D} \times N_{D D}} & \mathbf{0}_{N_{D D} \times N_{D D}} & \sigma_{\dot{\rho}}^{2} \boldsymbol{I}_{N_{s a t}}
\end{array}\right]
$$




\section{Multi-Receiver RTK}

In order to combine the measurements from multiple smartphones, their relative position inside the vehicle needs to be considered. To do so, a body frame is defined with the origin centered in one of the smartphones, called the master. Then, the positions of the other smartphones, called the slaves, are defined relative to the master smartphone. Three attitude angles are defined: pitch $\theta$, roll $\varphi$ and yaw $\psi$. The capacity to observe these attitude angles depends on the number and geometry of the smartphones in the car. In most of the campaigns the smartphones are aligned with the perpendicular axis of the vehicle, which does not allow the filter to observe the pitch angle. This, however, is not a problem for this project since the accuracy of the algorithm is evaluated in the horizontal error which is not very affected by an error in the pitch estimate. Figure 1 shows an example of a two-receiver geometry in the typical configuration that is found in the datasets. In this section, the master receiver is always indicated by the subscript 1 and the slave receivers are indicated by a subscript from 2 to $N_{r x}$.
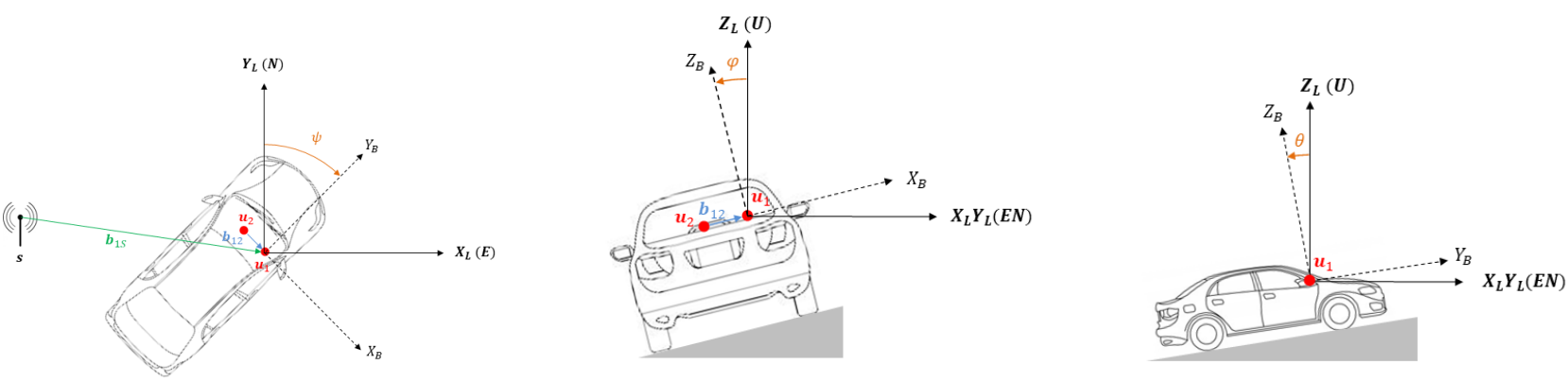

Figure 1: Multi-receiver geometry

The relative position of the slave receivers is then transformed from body frame to the ECEF frame. This transformation can be understood as a two-step operation. First, a transformation is made from body frame to Local Tangent Plane (LTP), expressed in ENU, and then a second transformation is made from LTP to ECEF. These two operations are made by the rotation matrices $C_{B 2 L}$ and $C_{L 2 E}$, respectively. For the 2-smartphone example, this is

$$
\boldsymbol{b}_{12}^{E}(\theta, \psi, \varphi)=\boldsymbol{C}_{L 2 E} \boldsymbol{C}_{B 2 L}(\theta, \varphi, \psi) \boldsymbol{b}_{12}^{B}
$$

where $\boldsymbol{b}_{12}^{E}$ and $\boldsymbol{b}_{12}^{B}$ are the baseline between master and slave smartphones expressed in ECEF and in body frame, respectively. Section 2.4 in [9] details how to build both rotation matrices. However, the rotation matrix from body to LTP is shown next as it will be necessary in the following section.

$$
\begin{aligned}
\boldsymbol{C}_{B 2 L}(\theta, \varphi, \psi) & =\left[\begin{array}{ccc}
\cos \psi & -\sin \psi & 0 \\
\sin \psi & \cos \psi & 0 \\
0 & 0 & 1
\end{array}\right]\left[\begin{array}{ccc}
\cos \varphi & 0 & \sin \varphi \\
0 & 1 & 0 \\
-\sin \varphi & 0 & \cos \varphi
\end{array}\right]\left[\begin{array}{ccc}
1 & 0 & 0 \\
0 & \cos \theta & -\sin \theta \\
0 & \sin \theta & \cos \theta
\end{array}\right] \\
& =\left[\begin{array}{ccc}
\cos \psi \cos \varphi & \cos \psi \sin \varphi \sin \theta-\sin \psi \cos \theta & \cos \psi \sin \varphi \cos \theta+\sin \psi \sin \theta \\
\sin \psi \cos \varphi & \sin \psi \sin \varphi \sin \theta+\cos \psi \cos \theta & \sin \psi \sin \varphi \cos \theta-\cos \psi \sin \theta \\
-\sin \varphi & \cos \varphi \sin \theta & \cos \varphi \cos \theta
\end{array}\right]
\end{aligned}
$$

\section{State transition model}

For this multi-receiver method, the attitude angles are included as state parameters, as well as the clock drifts and SD ambiguities of all receivers. Considering the example of two receivers, the state vector results in

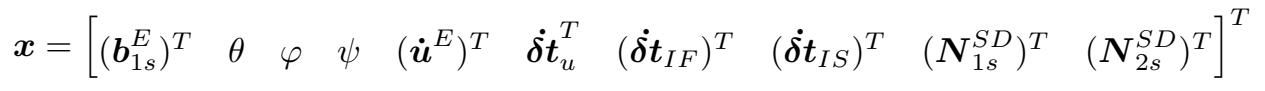

where $\boldsymbol{N}_{k s}^{S D}$ is the SD ambiguity vector for the $k$-th smartphone.

The state transition model is built very similarly to the single-receiver RTK. The user-station baseline is prediction is performed by integration of the velocity and the other state parameters follow a random walk model, including the three attitude angles 


$$
\begin{aligned}
\theta_{n} & =\theta_{n-1}+w_{\theta, n} \\
\varphi_{n} & =\varphi_{n-1}+w_{\varphi, n} \\
\psi_{n} & =\psi_{n-1}+w_{\psi, n}
\end{aligned}
$$

where

$$
w_{\theta, n} \sim \mathcal{N}\left(0, \sigma_{\theta}^{2}\right), w_{\varphi, n} \sim \mathcal{N}\left(0, \sigma_{\varphi}^{2}\right), w_{\psi, n} \sim \mathcal{N}\left(0, \sigma_{\psi}^{2}\right)
$$

Then, the state transition matrix can be built very similarly to the case of the single-receiver RTK in (10). This is, an identity matrix plus the time span $\delta t$ in the off-diagonal terms that relate the velocity with the relative position. The process noise covariance matrix is also very similar to the single-receiver case in (11) including the attitude variances in the diagonal.

\section{Measurement model}

Following the example of the two receivers, the measurement vector contains the DD measurements and the pseudorange rates from each receiver as

$$
\boldsymbol{z}_{n}=\left[\begin{array}{llllll}
\boldsymbol{\rho}^{\boldsymbol{D} \boldsymbol{D}_{1}^{T}} & \boldsymbol{\rho}_{2}^{\boldsymbol{D} \boldsymbol{D}_{2}^{T}} & \boldsymbol{\phi}^{\boldsymbol{D} \boldsymbol{D}_{1}^{T}} & \boldsymbol{\phi}_{2}^{\boldsymbol{D} \boldsymbol{D}_{2}^{T}} & \dot{\boldsymbol{\rho}}_{1}^{T} & \dot{\boldsymbol{\rho}}_{2}^{T}
\end{array}\right]^{T}
$$

The measurement model is defined such that the relative position of the master receiver with respect to the station is estimated. The measurement equation for the master receiver is defined as in the single receiver case of equations (12) and (13). Then, the measurement equations of the slave receivers are defined considering the baseline between smartphones as

$$
\begin{gathered}
\boldsymbol{h}_{\rho_{2}, n}(\boldsymbol{x})_{[j:]}=\left(\boldsymbol{e}_{s, p}-\boldsymbol{e}_{s, j}\right)^{T}\left(\boldsymbol{b}_{1 s}^{E}-\boldsymbol{b}_{12}^{E}\right) \\
=\left(\boldsymbol{e}_{s, p}-\boldsymbol{e}_{s, j}\right)^{T}\left(\boldsymbol{b}_{1 s}-\boldsymbol{C}_{L 2 E} \boldsymbol{C}_{B 2 L}(\theta, \varphi, \psi) \boldsymbol{b}_{12}^{B}\right) \\
\boldsymbol{h}_{\phi_{2}, n}(\boldsymbol{x})_{[j:]}=\left(\boldsymbol{e}_{s, p}-\boldsymbol{e}_{s, j}\right)^{T}\left(\boldsymbol{b}_{1 s}^{E}-\boldsymbol{b}_{12}^{E}\right)+\lambda N_{p}^{S D}-\lambda N_{j}^{S D} \\
=\left(\boldsymbol{e}_{s, p}-\boldsymbol{e}_{s, j}\right)^{T}\left(\boldsymbol{b}_{1 s}-\boldsymbol{C}_{L 2 E} \boldsymbol{C}_{B 2 L}(\theta, \varphi, \psi) \boldsymbol{b}_{12}^{B}\right)+\lambda\left(N_{p}^{S D}-N_{j}^{S D}\right)
\end{gathered}
$$

Equations (32) and (33) are clearly not linear due to the rotation matrix from body to LTP. Thus, they are linearized to obtain the measurement matrices as follows

$$
\begin{gathered}
\boldsymbol{H}_{\left.\rho_{2}^{D D}, n_{[} j:\right]}=\left[\begin{array}{lllllll}
\left(\boldsymbol{e}_{s, p}-\boldsymbol{e}_{s, j}\right)^{T} & h_{\theta, 2} & h_{\varphi, 2} & h_{\psi, 2} & 0 & \ldots & 0
\end{array}\right] \\
\boldsymbol{H}_{\left.\phi_{2}^{D D}, n_{[} j:\right]}=\left[\begin{array}{llllllll}
\left(\boldsymbol{e}_{s, p}-\boldsymbol{e}_{s, j}\right)^{T} & h_{\theta, 2} & h_{\varphi, 2} & h_{\psi, 2} & 0 & \cdots & 0 & \boldsymbol{\lambda}_{j}^{T}
\end{array}\right]
\end{gathered}
$$

where $\boldsymbol{\lambda}_{j}$ is a vector containing $\lambda$ and $-\lambda$ at the positions corresponding to the pivot and non-pivot satellites, respectively; and

$$
\begin{aligned}
h_{\theta, 2} & =\left(\boldsymbol{e}_{s, p}-\boldsymbol{e}_{s, j}\right)^{T} \boldsymbol{C}_{L 2 E}\left(\left.\frac{\partial}{\partial \theta}\right|_{\theta=\hat{\theta}} \boldsymbol{C}_{B 2 L}\right) \boldsymbol{b}_{1 k}^{B} \\
h_{\varphi, 2} & =\left(\boldsymbol{e}_{s, p}-\hat{\boldsymbol{e}}_{s, j}\right)^{T} \boldsymbol{C}_{L 2 E}\left(\left.\frac{\partial}{\partial \varphi}\right|_{\varphi=\hat{\varphi}} \boldsymbol{C}_{B 2 L}\right) \boldsymbol{b}_{1 k}^{B} \\
h_{\psi, 2} & =\left(\boldsymbol{e}_{s, p}-\boldsymbol{e}_{s, j}\right)^{T} \boldsymbol{C}_{L 2 E}\left(\left.\frac{\partial}{\partial \psi}\right|_{\psi=\hat{\psi}} \boldsymbol{C}_{B 2 L}\right) \boldsymbol{b}_{1 k}^{B}
\end{aligned}
$$


From equation (27), the partial derivatives of the $C_{B 2 L}$ with respect to the attitude angles $\theta, \varphi$ and $\psi$ are given by

$$
\begin{gathered}
\frac{\partial}{\partial \theta} \boldsymbol{C}_{B 2 L}=\left[\begin{array}{ccc}
0 & \cos \psi \sin \varphi \cos \theta+\sin \psi \sin \theta & -\cos \psi \sin \varphi \sin \theta+\sin \psi \cos \theta \\
0 & \sin \psi \sin \varphi \cos \theta-\cos \psi \sin \theta & -\sin \psi \sin \varphi \sin \theta-\cos \psi \cos \theta \\
0 & \cos \varphi \cos \theta & -\cos \varphi \sin \theta
\end{array}\right] \\
\frac{\partial}{\partial \varphi} \boldsymbol{C}_{B 2 L}=\left[\begin{array}{ccc}
-\cos \psi \sin \varphi & \cos \psi \cos \varphi \sin \theta & \cos \psi \cos \varphi \cos \theta \\
-\sin \psi \sin \varphi & \sin \psi \cos \varphi \sin \theta & \sin \psi \cos \varphi \cos \theta \\
-\cos \varphi & -\sin \varphi \sin \theta & -\sin \varphi \cos \theta
\end{array}\right] \\
\frac{\partial}{\partial \psi} \boldsymbol{C}_{B 2 L}=\left[\begin{array}{ccc}
-\sin \psi \cos \varphi & -\sin \psi \sin \varphi \sin \theta-\cos \psi \cos \theta & -\sin \psi \sin \varphi \cos \theta+\cos \psi \sin \theta \\
\cos \psi \cos \varphi & \cos \psi \sin \varphi \sin \theta-\sin \psi \cos \theta & \cos \psi \sin \varphi \cos \theta+\sin \psi \sin \theta \\
0 & 0 & 0
\end{array}\right]
\end{gathered}
$$

Regarding measurement model for the pseudorange rate, the attitude rate needs to be considered as the velocities may differ between receivers due to the lever arm. If the simple example of Fig 1 is considered, the velocity of the slave receiver can be expressed as a function of the velocity of the master receiver and the angular rates, $\omega_{z}$ and $\omega_{y}$, as

$$
\dot{\boldsymbol{u}}_{2}^{E}=\dot{\boldsymbol{u}}_{1}^{E}+\boldsymbol{C}_{L 2 E} \boldsymbol{C}_{B 2 L}\left\|\boldsymbol{b}_{12}\right\|\left[\begin{array}{c}
0 \\
\omega_{z} \\
\omega_{y}
\end{array}\right]
$$

The effect of the lever arm in the velocity of the slave receiver relative to the master receiver has been studied and compared to the noise of the Doppler measurements. To do so, two examples have been considered where two smartphones are located as in Figure 1 with a separation of 20 and 50 centimeters, which represent a normal and an worst case scenarios. Then, the distribution of the gyroscope measurements obtained in a urban area has been taken to compute the velocity error induced by the lever arm as in (40). This velocity is projected onto the LOS vectors of the example campaign so it can be compared to the Doppler measurement noise. As it can be seen in Figure 2, the error introduced by the lever arm in a worst-case scenario of 50 centimeters of separation falls inside the distribution of the Doppler noise. The error in the velocity introduced by the lever arm can then be considered as additional measurement noise by inflating the covariances of the Doppler measurements of the slave receivers.

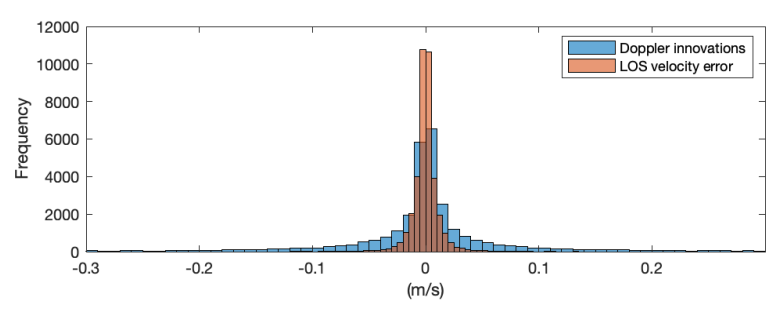

(a) $20 \mathrm{~cm}$ lever arm.

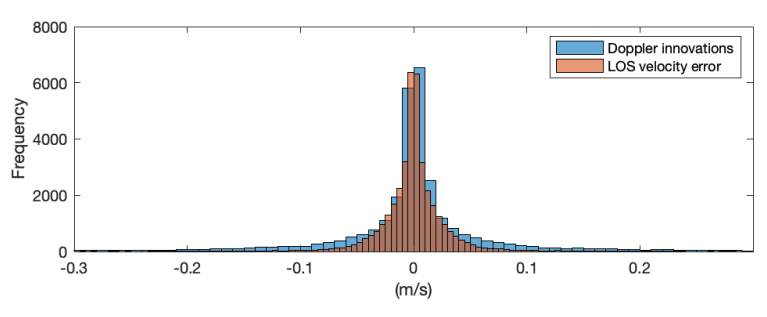

(b) $50 \mathrm{~cm}$ lever arm.

Figure 2: Distribution of pseudorange rate innovations (blue) and LOS velocity error induced by lever arm (red).

With this approximation, the velocities of the receivers are considered to be equal which considerably simplifies the equations and removes the dependency on the attitude rate in equation 40. Considering this approximation, the measurement matrix for the pseudorange rates is the same for the master and the slave receivers, which is obtained from the same model in (14) used for the SPPV algorithm.

$$
\boldsymbol{H}_{\left.\dot{\rho}_{2}, n_{[} i:\right]}=\left[\begin{array}{llllllll}
\mathbf{0}_{1 \times 3} & \mathbf{0}_{1 \times 3} & \left(\boldsymbol{e}_{s, p}-\boldsymbol{e}_{s, j}\right)^{T} & \boldsymbol{a}_{i}^{T} & \boldsymbol{b}_{i}^{T} & 0 & \ldots & 0
\end{array}\right]
$$


Finally, the measurement matrix for the example of two receivers is

$$
\boldsymbol{H}_{n}=\left[\begin{array}{c}
\boldsymbol{H}_{\rho_{1}^{D D}, n} \\
\boldsymbol{H}_{\rho_{2}^{D D}, n} \\
\boldsymbol{H}_{\phi_{1}^{D D}, n} \\
\boldsymbol{H}_{\phi_{2}^{D D}, n} \\
\boldsymbol{H}_{\dot{\rho}_{1}, n} \\
\boldsymbol{H}_{\dot{\rho}_{2}, n}
\end{array}\right]
$$

In this method, the measurements of the different smartphones are processed by the EKF at the time they are available. This assumes that the clocks of the smartphones are synchronized, which is plausible considering that the smartphones are connected to the Internet and they periodically steer their clocks to match with UTC time. However, this synchronization may not be perfect and in that case an additional error would be added to the estimates due to an inter-smartphone clock bias. In order to solve this problem, the inter-smartphone clock bias could be added in the model as an extra state parameter.

\section{Outlier rejection}

One way to mitigate the impact of observation outliers, is to use the information the innovation covariance provides about the expected distribution of innovations, to decide whether a given innovation looks like an outlier, i.e. is unlikely to be a realization of this distribution, and in this case to exclude it from the filter update. In this project, a standard hypothesis testing applied to the innovations is implemented [5]. This method also provides the capability of cycle-slip detection in regards of the carrier phase measurements, since these cycle-slips generate also outliers in the carrier phase measurements. However, depending on the state covariance, small cycle slips will probably not be detected. For this reason it would be interesting to implement some dedicated cycle slip detection method in the future.

As an example, Figure 3 shows a comparison of the pseudorange innovations obtained with the single-receiver RTK using no outlier rejection against the same method with the outlier rejection activated and set to a probability of false outlier rejection equal to 0.1. When no outlier rejection is activated, the pseudorange innovations show the large amount of outliers found in the Android GNSS measurements. These outliers actually cause the position estimates to drift so much that the estimation stops around epoch 1495 because all the satellites are below the elevation mask with respect to the last position estimated. On the contrary, when activating the outlier rejection, all the innovations fall within normal values and the position estimate accuracy remains around the 10 meters.

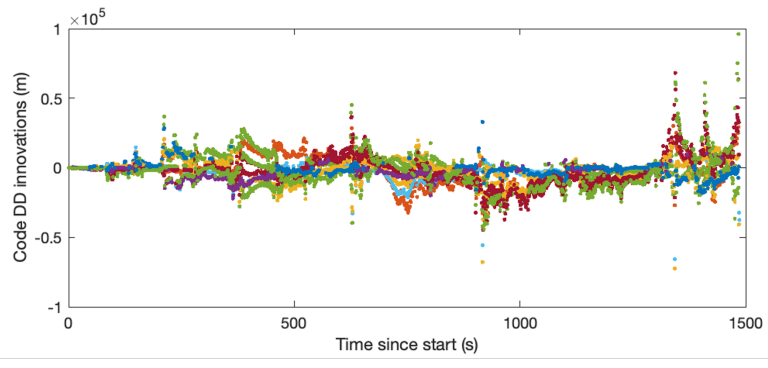

(a) No outlier rejection

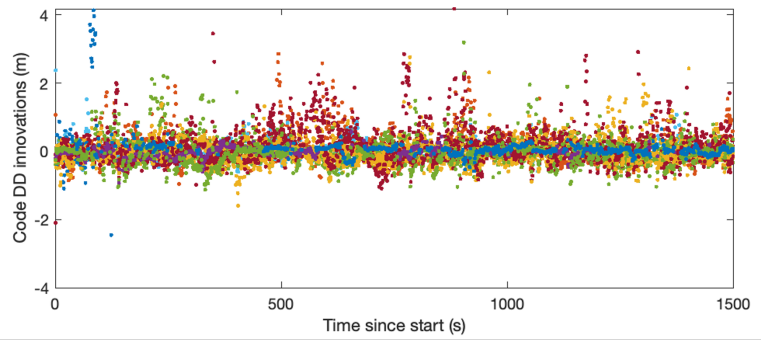

(b) Probability of False Outlier Rejection $=0.1$.

Figure 3: Pseudorange innovations from single-receiver RTK for trace 2021-04-28-US-SJC-1_Pixel4.

\section{MEASUREMENT ANALYSIS}

The organizers of the Google Smartphone Decimeter Challenge have provided the Android GNSS Raw measurements and the INS measurements in text files called GnssLog, which follow a similar format as CSV files. These files provide GNSS measurements taken at a constant rate of $1 \mathrm{~Hz}$. As well as the Android Raw GNSS measurements, the organizers of the competition have also provided the measurements in RINEX files. These files, however, do not contain all the information that is included in the raw measurement files, in particular the uncertainties related to the GNSS measurements. RINEX files, however, are simpler to use since parsers are readily available as part of existing software. In order to evaluate which source is better to use, the GNSS observables obtained from preprocessing the raw measurements have been compared to the ones obtained from the RINEX files. A summary of this comparison is provided in this section. Then, an analysis of the reliability of the uncertainties provided by the GnssLog files is also given. In order to perform these analyses, a standalone SPP EKF 
implementation was used to estimate the clock bias, the measurement residuals and to provide an assessment of the performance that could be obtained from the provided data.

\section{Calculation of GNSS observables}

The main particularity of the Android Raw GNSS measurements is that they do not provide the pseudorange measurements directly, instead, Android provides the necessary parameters to compute them. These parameters include the internal hardware clock, the time of transmission of the signal and the bias of the receiver clock with respect to the reference GNSS system. While the reception time is obtained in the GNSS system time, the transmission time is referenced to the constellation of each measurement. Therefore, both times have to be set to the same reference before computing the time difference required to obtain the pseudoranges. In this project, measurements from up to three different GNSS constellations are used, namely the US-American GPS, the European Galileo and the Chinese BeiDou. Measurements from the Russian system GLONASS is also provided in most of the measurement files. However, since GLONASS is implemented over Frequency-Division Multiple Access, the use of Doppler measurements and the implementation of RTK becomes more complex. Therefore, this constellation was omitted in the execution of this project.

Following the steps described in section 2.4 of GSA's white paper [10], the calculation of the pseudoranges can be summarized as

$$
\rho=\frac{t_{R x}-t_{T x}}{10^{9}} c
$$

where $t_{T x}$ and $t_{R x}$ are the transmission and reception times, respectively. The transmission time is obtained directly from the parameter ReceivedSvTimeNanos and it is referred to the system time of the constellation of the measurement. The reception time is computed from the hardware clock timestamp and the bias to the true GPS time. However, it is referred to the system time of the smartphone (generally GPS). The calculation of the reception time in GPS time can be summarized as the following expression

$$
t_{R x}^{G}=t_{\text {clock }}-t_{\text {bias }}
$$

Equation (44) is actually a simplification of the complete calculation which is fully detailed in [10]. The resulting time, $t_{R x}^{G}$ is then shifted so that it is referenced to the system time of the measurement, which provides the transmission time used in equation (43).

The raw parameters include uncertainties for the receiver clock time, $\sigma_{t_{c l o c k}}$, the receiver clock bias, $\sigma_{t_{b i a s}}$, and the transmission time, $\sigma_{t_{T x}}$. Then, the standard deviation of the pseudorange measurement can be estimated as

$$
\sigma_{\rho}=c \sqrt{\sigma_{t_{c l o c k}}^{2}+\sigma_{t_{b i a s}}^{2}+\sigma_{t_{T x}}^{2}}
$$

As per the carrier phase and the Doppler measurements, the first one can be directly obtained from the Accumulated Delta Range (ADR) which is provided in meters, and the second one is provided as the pseudorange rate in meters per second. Both these measurements have their associated uncertainties expressed in meters and meters per second, respectively. Therefore, they can be directly used as an estimate of the standard deviations of the measurements.

\section{Comparison of GNSS measurement sources}

In order to verify the preprocessing of GNSS observables from the raw measurements and compare them to the measurements from RINEX, the GnssAnalysis software was used. GnssAnalysis is a Desktop application developed by Android which allows the user to analyze the GNSS measurements obtained with a smartphone. Apart from a set of plots, this application can also provide a file with the preprocessed measurements. However, the code of this application is not accessible so it could not be used in our project to automate the computation of GNSS observables. Nevertheless, it could be used to obtain the observables of a single trace and compare them to the results of our preprocessing and the observables obtained from the RINEX. Figure 4 shows an example of the differences between the measurements generated by our preprocessing step with the measurements obtained from the RINEX files and the GnssAnalysis application. It can be seen that our calculations provide almost the same result as the GnssAnalysis for all the measurement types, with differences of half a millimeter on the pseudoranges or one hundredth of a cycle (less than $2 \mathrm{~mm}$ in L1) in the phase. However, the pseudoranges largely differ when compared to the ones obtained from the RINEX. 

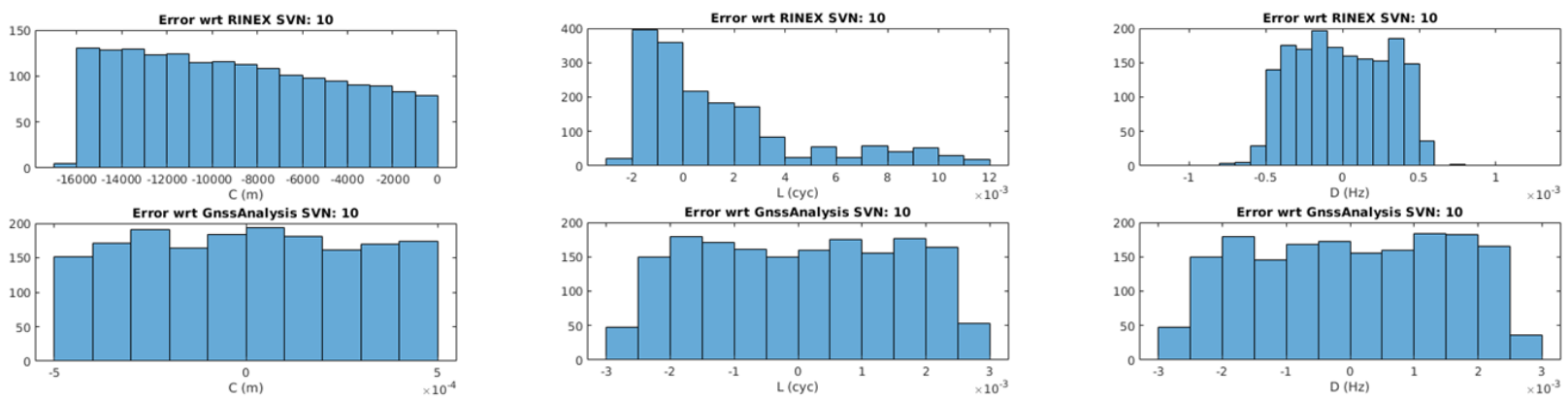

Figure 4: Differences of the preprocessed measurements of a single satellite compared to the RINEX (top) and the GnssAnalysis (bottom). From left to right: pseudorange (meters), carrier phase (cycles) and Doppler (Hz).

Figure 5 shows a comparison of the pseudorange residuals obtained from the WLS processing the RINEX and the preprocessed raw measurements. It can be seen that the residuals from the first source follow a bi-modal distribution, which is unexpected in the pseudorange measurements, as the noise is expected to be approximately zero-mean.

Finally, the differences between the pseudoranges obtained from the RINEX and the ones obtained by processing the GnssLog are computed and it matches with the receiver clock bias computed with the WLS method. Figure 6 shows this comparison for a single satellite as an example. This shows that in the generation of the RINEX files from the raw measurements, the receiver clock bias has been corrected in the pseudorange measurements. However, it is not corrected in the carrier phase measurements, since these measurements are almost the same between sources (Fig. 4). Although this difference could be solved by keeping two different estimations of the clock bias, it only makes the implementation more complex and prone to errors. For this reason, it was decided to use the GnssLog files as the source for the GNSS measurements.
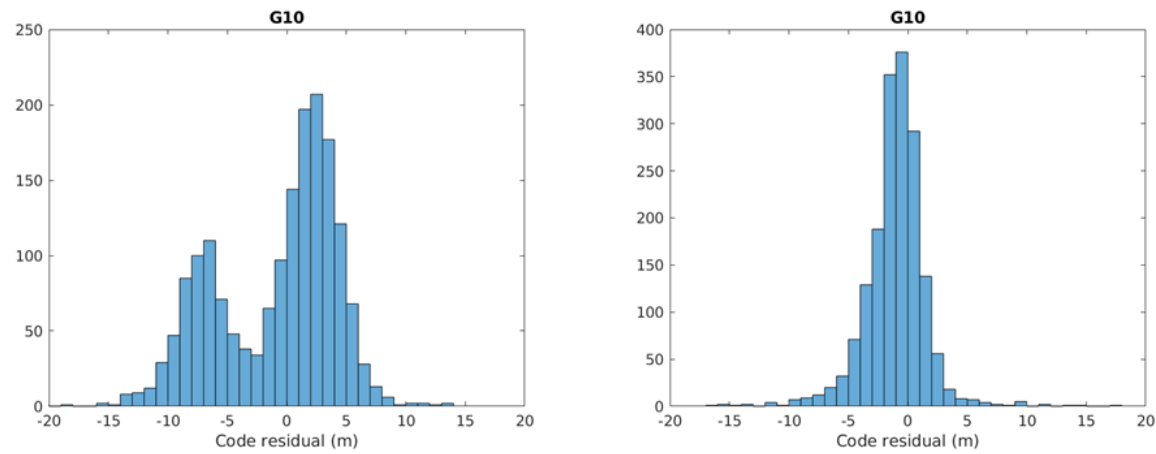

Figure 5: Distributions of the pseudorange residuals obtained from the RINEX (left) and the preprocessed raw measurements (right).
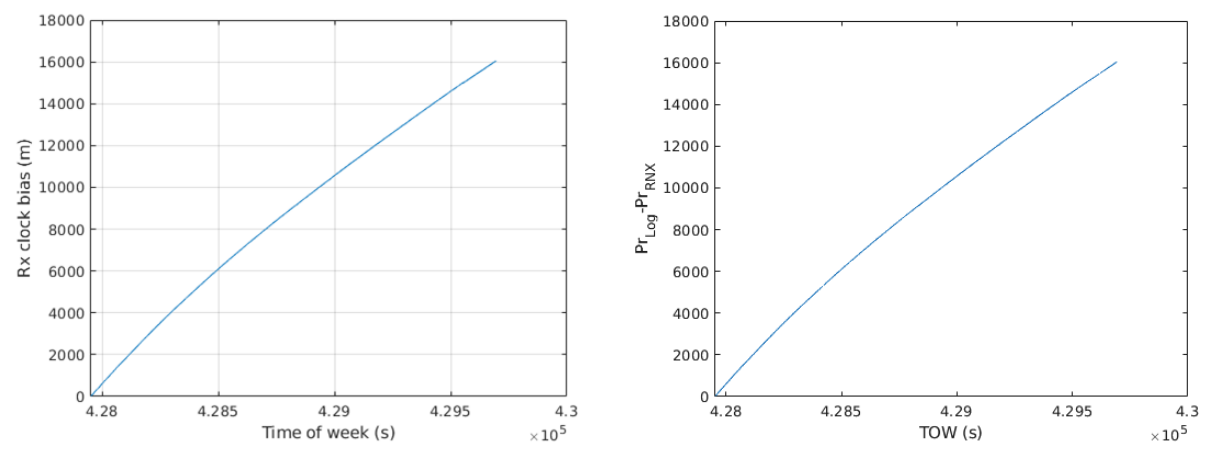

Figure 6: Receiver clock bias estimated by the WLS (left) and difference of pseudorange between RINEX and GnssLog (right). 


\section{Reliability of the measurement uncertainties}

One of the particularities of the Android GNSS raw measurements is that an uncertainty of the measurement is provided for each GNSS observation. However, the Android documentation does not detail how these uncertainties are obtained. To determine the reliability of the uncertainties, the standard deviation of the pseudoranges is computed from these uncertainties as in equation (45) and it is compared to the pseudorange residuals obtained with the standalone SPP EKF. Figure 7 shows the moving standard deviation of the pseudorange residuals plotted against the moving mean of the standard deviation calculated from the uncertainties, which have been computed from 1700 epochs of measurements of 9 satellites. In comparison, the same plot is shown for the standard deviation computed as follows

$$
\hat{\sigma}_{\rho, i}=\frac{\sigma_{\rho, 0}}{\sin \left(\alpha_{i}\right)}
$$

where $\hat{\sigma}_{\rho, i}$ is the estimate of the pseudorange standard deviation for satellite $i, \sigma_{\rho, 0}$ is a default value set to 3 meters and $\alpha_{i}$ is the elevation of the $i$-th satellite.
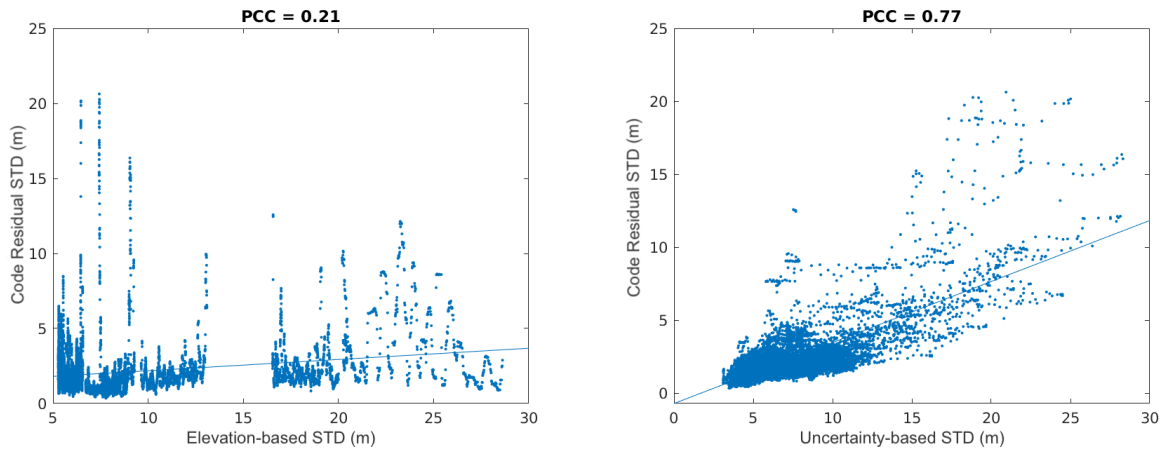

Figure 7: Pseudorange residuals vs elevation-based standard deviation (left). Pseudorange residuals vs uncertainty-based standard deviation (right).

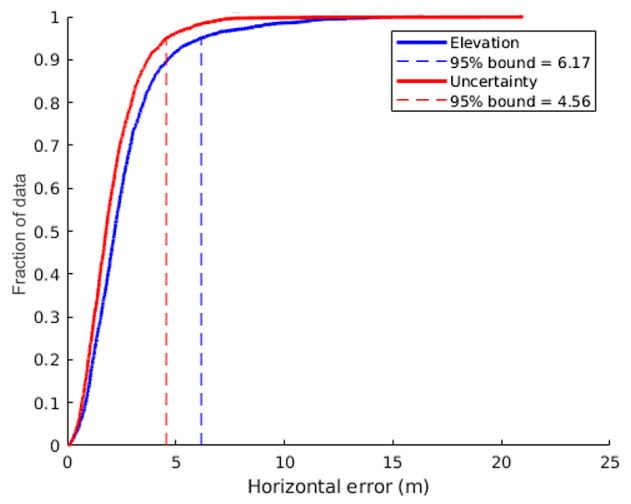

Figure 8: CDF of horizontal error from SPP EKF using elevation-based variance (blue) vs uncertainty-based variance (red).

It can be seen that the uncertainty-based standard deviation shows a high correlation with the noise in the measurements, with a Pearson Correlation Coefficient (PCC) close to 0.8. In contrast, the typical elevation-based estimation of the standard deviation shows no correlation with the actual noise in the measurements. Therefore, the uncertainties provided by Android seem to be much more reliable than the elevation-based model for estimating the measurement variance. For further evidence, Fig. 8 shows the Cumulative Distribution Functions (CDF) of the horizontal error of the standalone SPP KF estimation using the two different pseudorange variance estimations. It can be seen that the uncertainty-based variance provides better results, with a $95 \%$ accuracy of $4.56 \mathrm{~m}$ versus 6.17 from the elevation-based model. Thus, the measurement covariance matrices of all the methods used in this project are built using the uncertainties provided in the Android GnssLog files. 


\section{RESULTS}

In this section, the proposed multi-receiver RTK method is verified with the train dataset measurements. Results for the test dataset are not shown because no reference is available to analyze the performances. In order to provide an analysis of the performance of the multi-receiver RTK on individual trajectories, two measurement campaigns are chosen. The first campaign that is used is 2020-05-29-US-MTV-1, which corresponds to an open-sky scenario which contains measurements from 3 smartphones (namely Pixel4, Pixel4XL and Pixel4XLModded) taken on a highway next to Mountain View, California. The second campaign is 2021-04-28-US-SJC-1, which corresponds to an urban scenario with measurements from 2 smartphones (namely Pixel4 and SamsungS20Ultra) taken in the streets of San Jose, California. Additionally, this section also provides an overview of the results obtained on all of the measurement campaigns for both the single- and the multi-receiver RTKs.

\section{Comparison between positioning methods}

In the following, the performance of the three methods explained in section II is compared. Additionally, the results obtained with the conventional SPP and SPPV EKFs are provided in order to observe the effect of using the Doppler measurements and to show the advantage of implementing RTK for positioning with smartphones.

The trajectories estimated by these four positioning methods are shown in Figures 9a and $9 \mathrm{~b}$ for the highway and the urban scenarios, respectively. In order to study the accuracy of the four different methods, Figure 10 shows the CDFs and 95 percentiles of the horizontal errors in the highway and the urban campaigns.

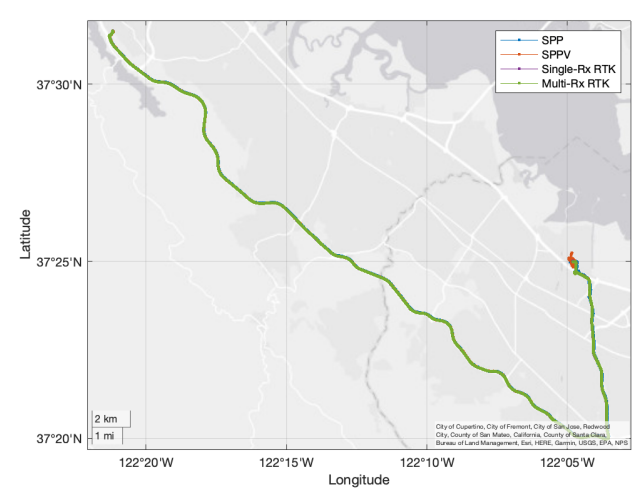

(a) 2020-05-29-US-MTV-1

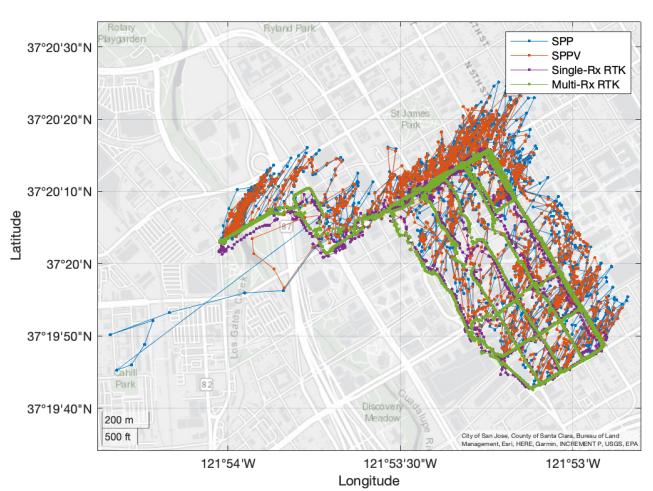

(b) 2021-04-28-US-SJC-1

Figure 9: Estimated positions with SPP, SPPV, single-receiver RTK and multi-receiver RTK.

As it is expected, the accuracy of the positions is much better in the highway campaign, since the measurements are much less affected by multipath and there is less signals are obstructed, which results in better satellite geometry. Also, it can be seen that the RTK performs better than the SPP and SPPV methods thanks to the use of differential corrections. However, it is interesting to see that the main improvement in the highway campaign comes from the inclusion of the Doppler measurements. A hypothesis for this big improvement is that the inclusion of Doppler measurements in the EKF provide an indirect smoothing of the pseudorange measurements, since the Doppler is less affected by noise. This is especially significant when the measurements are taken by smartphones, since the noise is higher due to the miniaturized and low-cost hardware. On the urban scenario, however, the main improvement comes by far with the RTK method. This is also surprising because one would expect that the error in the urban scenario would be more driven by the multipath, which is not corrected on the differential measurements.

\section{Accuracy of multi-receiver RTK}

In order to study the effect of combining measurements from multiple receivers, the same two campaigns used in the previous section are taken as an example. In both campaigns, the positions of all smartphones are computed using the single-receiver RTK. The results are compared to the multi-receiver RTK, which combines all the measurements of that campaign. Figure 11 shows the horizontal error CDFs for the two campaigns mentioned. In both cases, the error at the 95 percentile is lower with the multi-receiver method than using any of the single receivers. However, as it can be seen in the lower percentiles, this better performance of the multi-receiver RTK is not consistent during all the trajectory. For these tests, the measurement covariances of different smartphones were not weighted differently. However, a different weight could be given to the measurements of each smartphone according to the observed accuracy in the single-receiver method or on the measurements residuals, which could improve the performance of the multi-receiver RTK. 


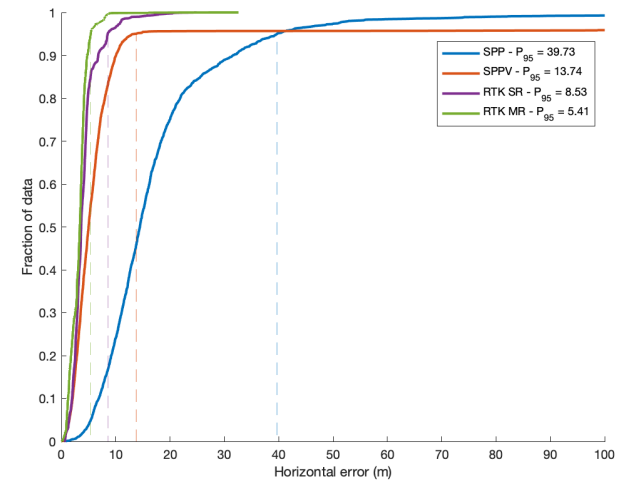

(a) 2020-05-29-US-MTV-1

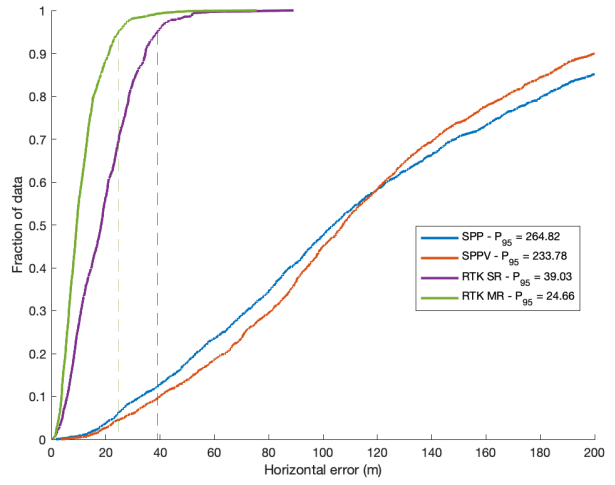

(b) 2021-04-28-US-SJC-1

Figure 10: Horizontal error CDFs of SPP, SPPV, single-receiver RTK and multi-receiver RTK.



(a) 2020-05-29-US-MTV-1

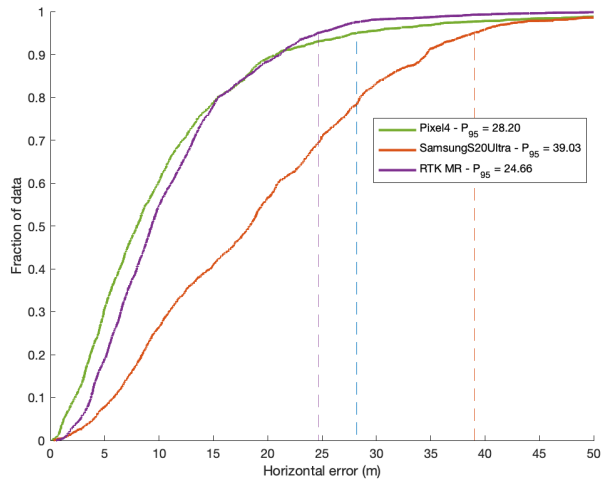

(b) 2021-04-28-US-SJC-1

Figure 11: Comparison between single-receiver and multi-receiver.

The results obtained with the single-receiver methods described in this project (namely SPPV and RTK) have shown a largely varying accuracy on different traces. It has been observed that the evaluation of some traces provided errors of several hundreds of meters while other traces from the same campaign showed errors on the order of the few meters. However, these large errors were not always associated to the same smartphone model. An analysis has been made to try to identify the causes of the worst scores, however they have not been identified and this problem has been left for future work. In order to continue with the analysis, the measurements that resulted in scores over 50 meters have been omitted in the the multi-receiver method.

Figure 12 shows the scores obtained using both the single- and the multi-receiver RTK methods on all the measurement campaigns provided in the train dataset. The blue and the orange bars denote the single- and the multi-receiver solutions, respectively. Red bars indicate the smartphones that have not been used in the multi-receiver solution on that campaign. It can be seen that the multi-receiver solution generally improves the performance of the single-receiver ones. Although this improvement is only of the order of the centimeters in some campaigns (e.g. campaign 1, 4 and 10), the score of the multi-receiver improves by around $32 \%$ with respect to the best single-receiver score in campaign 5 and by more than $25 \%$ in campaign 6 . However, in three of the campaigns $(15,17$ and 20), the accuracy of the estimate is reduced with the multi-receiver method. This problem may be solved by a proper weighting of the different smartphones and/or the estimation of an inter-smartphone clock bias and it has been left for future work. 


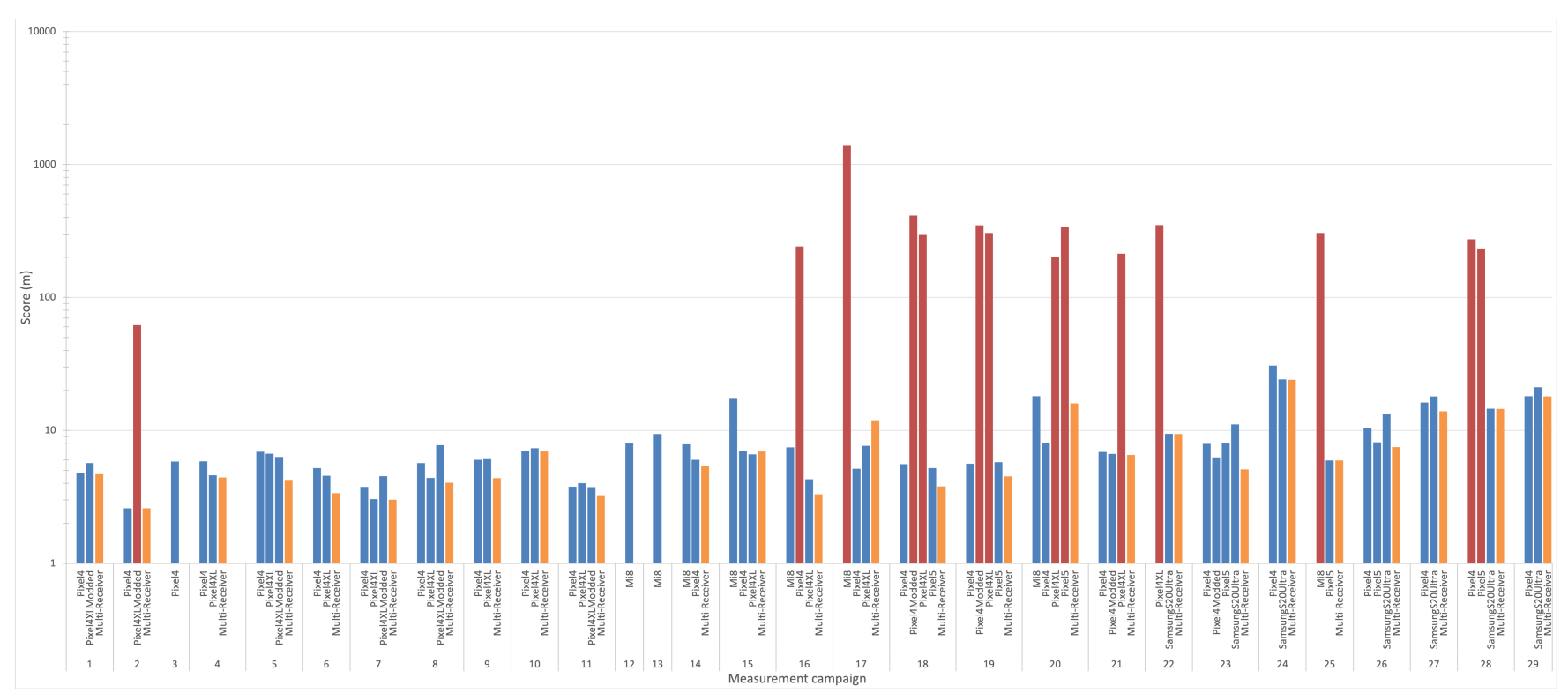

Figure 12: Single- and multi-receiver RTK scores for all traces in the train dataset. Blue denotes single-receiver file used in the multi-receiver RTK and red denotes single-receiver file omitted in the multi-receiver RTK.

\section{CONCLUSION}

This paper presented a method to combine GNSS measurements from multiple Android smartphones with known geometry which provides attitude estimation and enhances the performance of RTK. Additionally, the use of Doppler shift and carrier phase measurements is studied in the context of positioning using smartphones. Results from four different techniques have been shown in this article. The first technique is a standalone Single Point Position implemented with an EKF that is used as a base reference. The second technique uses an Extended Kalman Filter to combine pseudorange and Doppler measurements, which provides an estimation of the position and velocity of the receiver. It has been shown that the inclusion of Doppler measurements notably improves the accuracy of the estimates. The third technique consists of an RTK with float ambiguity resolution, which also includes non-differential Doppler measurements to estimate the velocity. The use of differential GNSS measurements has shown an improvement in the performance of the estimation. However, it has been seen that the inclusion of carrier phase measurements does not always provide the expected improvements, probably due to the lower quality of these measurements on smartphones. We think that this problem could be tackled with a more sophisticated outlier rejection mechanism and a dedicated cycle slip detection method. Finally, the third technique combines simultaneous measurements from different smartphones in order to improve the performance of the RTK and also provide an estimation of the attitude. This technique assumes that the relative positions of the smartphones in the vehicle are known. This, however, is not the case for the test dataset used for evaluation in this competition. Thus, this paper only shows the results on the train dataset, for which the geometries have been obtained from the ground-truth files. It is shown that the multi-receiver technique improves the accuracy of the estimates in the majority of the situations.

We have seen that in some cases the multi-receiver estimates are worse than some of the single-receiver ones. To solve this, two enhancements are proposed. The first and simpler one refers to the weighting of the measurements from different smartphones, since in this article the different qualities of the measurements between smartphones is not considered. For that, it could be interesting to research on the possibility of automatically weighting the different receivers from the data included in the raw measurements such as the measurement uncertainties. The second enhancement refers to the synchronization between smartphones. We have assumed that the smartphones are more or less synchronized since they are connected to the Internet and the clock is periodically corrected. However, an inclusion of an inter-smartphone clock bias could be studied to improve the performance of the multi-receiver RTK.

Additionally, some other matters have been left for future research due to the limited amount of time in the competition. Firstly, it would be interesting to further research on the nature of the Android GNSS measurements, especially on the use of the carrier phase since it has not provided the improvement in performance that can be observed in dedicated GNSS receivers. Secondly, the implementation of an integer ambiguity resolution method, such as LAMBDA, is expected to improve the performance of the estimation and it would complete the implementation of the RTK. Furthermore, the inclusion of a dedicated cycle slip detection method is expected to provide even better performance when using carrier phase measurements. Thirdly, the use of a 
non-diagonal measurement covariance matrix on the RTK methods should improve the performance of the estimates. Although the workaround implemented in this project still provides acceptable accuracies, it would be more rigorous to include the non-diagonal terms and provide a better noise model to the Kalman Filter. Also regarding the multi-receiver technique, it has been seen that the calibration of the smartphones geometry is important to provide a good positioning accuracy. Therefore, the implementation of a calibration step could also notably improve the performance of the multi-receiver RTK. To do so, some estimation technique could be implemented, such as a Least Squares, which estimates the geometry of the receivers from the GNSS measurements, considering that the geometry is constant during all the trajectory. Finally, the hybridization of the RTK methods with INS and magnetometer measurements would reduce the covariance of the estimates because a more accurate transition model would be used. This could also improve the accuracy of the estimates, although it would require to deal with the problems of non synchronization and outages of the INS measurements.

\section{ACKNOWLEDGEMENTS}

This study has been possible thanks to the Android GNSS raw measurements published by Google for the Google Smartphone Decimeter Challenge as well as the Verizon Hyper-Precise Location RINEX Base-Station Files released by Yahoo and available in https://webscope. sandbox.yahoo.com/.

Any acknowledgements should appear just before the references section at the END of the paper.

\section{REFERENCES}

[1] G. M. Fu, M. Khider, and F. van Diggelen, "Android raw gnss measurement datasets for precise positioning," in Proceedings of the 33rd International Technical Meeting of the Satellite Division of The Institute of Navigation (ION GNSS+ 2020), 2020, pp. 1925-1937.

[2] X. Hu, P. Thevenon, and C. Macabiau, "Attitude determination and RTK performances amelioration using multiple lowcost receivers with known geometry," in Proceedings of the 2021 International Technical Meeting of The Institute of Navigation, 2021, pp. 439-453.

[3] — - "Cycle slip detection and repair using an array of receivers with known geometry for RTK positioning," in 2020 IEEE/ION Position, Location and Navigation Symposium (PLANS), 2020, pp. 1123-1134.

[4] — , "Improvement of RTK performances using an array of receivers with known geometry," in Proceedings of the 2020 International Technical Meeting of The Institute of Navigation, 2020, pp. 440-453.

[5] J.-G. Wang et al., "Test statistics in kalman filtering," Positioning, vol. 1, no. 13, 2008.

[6] E. D. Kaplan and C. Hegarty, Eds., Understanding GPS: principles and applications, 2nd ed., ser. Artech House mobile communications series. Boston: Artech House, 2006, oCLC: ocm62128065.

[7] D. Simon, Optimal state estimation: Kalman, H infinity, and nonlinear approaches. John Wiley \& Sons, 2006.

[8] Peter S. Maybeck, Stochastic models, estimation and control. Volume 1, 1st ed. New York: Academic press, 1979 , vol. 1.

[9] P. D. Groves, Principles of GNSS, Inertial, and Multi-sensor Integrated Navigation Systems, 1st ed. Artech House, 2008, vol. 39. [Online]. Available: https://www.emerald.com/insight/content/doi/10.1108/ir.2012.04939caa.011/full/html

[10] European GNSS Supervisory Authority., Using GNSS raw measurements on Android devices: white paper. LU: Publications Office, 2017. [Online]. Available: https://data.europa.eu/doi/10.2878/449581 